\title{
Pronóstico de Reducción de Emisiones, de Enfermos y de Gastos Asociados al incluir el Gas Natural dentro de la Matriz Energética en Perú
}

Freddy J. Rojas ${ }^{(1) \star}$, Sergio Pacsi(2), Odón R. Sánchez-Ccoyllo(3), y Milka M. Perales ${ }^{(4)}$

(1) Facultad de Ingeniería, Sec. Ing. Mecánica, Pontificia Universidad Católica del Perú, Av. Universitaria 1801, San Miguel, Lima-Perú (fjrojas@pucp.edu.pe)

(2) Facultad de Ciencias, Ingeniería Ambiental, Universidad Nacional Agraria La Molina, Av. La Molina s/n,

La Molina, Lima-Perú (spv@lamolina.edu.pe)

(3) Carrera profesional de Ingeniería Ambiental, Universidad Nacional Tecnológica de Lima Sur, Av. Central,

Villa EL Salvador, Lima-Perú (osanchezbr@gmail.com)

(4) Facultad de Ingeniería, Sec. Ing. Industrial, Pontificia Universidad Católica del Perú, Av. Universitaria 1801,

San Miguel, Lima-Perú (milka.perales@pucp.pe)

* Autor a quien debe ser dirigida la correspondencia.

Recibido Ago. 14, 2018; Aceptado Oct. 29, 2018; Versión final Dic. 18, 2018, Publicado Jun. 2019

\section{Resumen}

Este trabajo presenta el estudio de la reducción de los contaminantes atmosféricos $\left(\mathrm{MP}_{10}, \mathrm{MP}_{2.5}, \mathrm{CO}, \mathrm{SO}_{2}\right.$ y $\mathrm{NO}_{\mathrm{x}}$ ) por el uso del gas natural. Se analiza el impacto en el sector salud (reducción de enfermos por vías respiratorias), en el sector económico (reducción de costo de inasistencia laboral y costo por atención por salud), en Lima Metropolitana al 2040. Se realizó un estudio detallado del parque automotor considerando el ingreso del gas natural en la matriz energética desde el año 2004, de enfermos por contaminación atmosférica, gastos asociados a la salud de las personas, luego se realizaron cálculos de emisiones mediante los factores de emisión como herramienta principal en este estudio y se realizaron los pronósticos mediante los cálculos estadísticos al 2040. Se concluyó con una propuesta de inversión a la conversión del parque automotor hacia el gas natural consiguiendo favorablemente la reducción de enfermos por vías respiratorias, de costo por salud para el estado, y reducción de la contaminación atmosférica.

Palabras clave: contaminación atmosférica; enfermos; factores de emisión; salud; gas natural.

\section{Forecast of Reduction of Emissions, Patients, Associated Expenses using Emission Factors and considering Natural Gas within the Energy Matrix in Peru}

\begin{abstract}
The progress of the study on the estimation and forecast of air pollution in Lima-Peru is presented and discussed. This work presents the study of the reduction of atmospheric pollutants $\left(\mathrm{PM}_{10}, \mathrm{MP}_{2.5}, \mathrm{CO}, \mathrm{SO}_{2}\right.$ and NOx), reduction of patients, cost reduction due to health, due to the use of natural gas in Metropolitan Lima by 2040. A detailed study of the vehicle fleet considering the entry of natural gas into the energy matrix since 2004 , of patients due to atmospheric pollution, expenses associated with people's health, then emission calculations were carried out using the emission factors as the main tool in this study and forecasts were made by statistical calculations to 2040. It was concluded with an investment proposal to the conversion of the automotive fleet to natural gas getting favorably the reduction of patients, cost for health for the state, and reduction of air pollution.
\end{abstract}

Keywords: Air pollution; sick; emission factors; health; natural gas. 


\section{INTRODUCCIÓN}

La contaminación atmosférica es uno de los problemas ambientales más importantes que afectan a nuestro mundo, como resultado de la presencia en el aire de materiales nocivos producidos por el hombre, en cantidades grandes como para producir efectos perjudiciales para el medio ambiente como para el hombre. Dicho problema, está presente en todas las sociedades, independientemente del nivel de desarrollo socioeconómico, y constituye un fenómeno que tiene particular incidencia sobre la salud del hombre (Placeres et al., 2006), es un problema de las actividades humanas (antropogénico) y más aún en las sociedades con mayor poder económico debido a que consumen más energía y producen mayor cantidad de emisiones, como por ejemplo la exposición de partículas finas como el PM2.5 que contribuyen a las enfermedades respiratorias en la India (Conibear et al., 2018), en otro estudio se determinó como el exceso en la mortalidad y morbilidad es debido al PM10 que los gases contaminantes (Maji et al., 2016). Según la Organización Mundial de la Salud (OMS) en las "Guías de calidad de aire" mencionan que los cuatro contaminantes que ocasionan perjuicio a la salud son: material particulado (PM, por sus siglas en inglés), ozono (O3), dióxido de nitrógeno (NO2) y dióxido de azufre (SO2).

La OMS menciona para el 2012 hubo 3 millones de muertes prematuras por año, de las cuales el $72 \%$ murió por cardiopatía isquémica y accidente cardiovascular, el 14\% por neumonía obstructiva crónica o infección aguda de las vías respiratorias inferiores y el $14 \%$ murió de cáncer pulmonar debido a la exposición principal de PM10 o menores. Según, el (Banco Mundial, 2007), para el 2005 Lima lideraba como la ciudad más contaminada con más de $80 \mu \mathrm{g} / \mathrm{m} 3$ anual de PM10. Además, la Dirección de Salud V "DISA V" (Minsa, 2012), manifiesta estadísticamente que la morbilidad en Lima Metropolitana en el año 2006 y 2007 se debe por afecciones respiratorias en un $25 \%$. Esto demuestra que tenemos una ciudad muy contaminada por el uso de los combustibles tradicionales. Como antecedente a nivel normativo, en el año 2001, se aprobó el Reglamento de Estándares Nacionales de Calidad Ambiental del Aire, mediante el Decreto Supremo 0742001-PCM (norma publicada el 24 de junio del 2001), en el cual se restringe a los contaminantes Dióxido de Azufre, Dióxido de Nitrógeno, Ozono, PM10 y PM2.5.

Posteriormente, en el año 2003, se aprobó el Reglamento de los Niveles de Estados de Alerta Nacionales para Contaminantes del Aire, a través del Decreto Supremo $N^{\circ} 009-2003-S A$ (norma publicada el 25 de junio del 2003) el cual tiene como objetivo controlar la contaminación aguda a través de exposiciones de corta duración. Esta norma fue posteriormente modificada a través del Decreto Supremo 003-2017-MINAM (publicada el 07 de junio del 2017). Según el Ministerio del Ambiente (2012) los principales responsables de la contaminación en Lima Metropolitana se encuentran generados por el parque automotor (70\%) y el porcentaje restante por la industria estacionaria (MINAM, 2012). Según SENAMHI (2012) entre las especies químicas más frecuentes que causan alteraciones en la composición de la atmósfera se encuentran los aerosoles, óxidos de azufre (SOx), monóxido de carbono (CO), óxidos de nitrógeno (NOx), hidrocarburos $(\mathrm{HnCm})$, ozono (O3) y dióxido de carbono (CO2).

En el este de China se realizó una actualización del inventario de emisiones debido a que este es una herramienta fundamental para el estudio de pronóstico de contaminación atmosférica (Zhang et al., 2018). En un trabajo de investigación de modelamiento de emisiones vehiculares en las ciudades de China mencionan que las emisiones están relacionadas al tipo de vehículo y combustible usado en la ciudad. Menciona algo muy importante relacionado a que los factores de emisión por ciudad son diferentes y esto es debido a las características locales de tráfico en cada ciudad (Huo et al., 2011). En Colombia en un estudio con los factores de emisión se determinó la estimación de contaminantes atmosféricos provenientes de fuentes móviles encontrando que los autos son los que más aportan los niveles de contaminación de CO (Londoño et al., 2011). También, Wang et al. (2008) realizaron un estudio similar en Shanghái-China, obteniendo que el tráfico vehicular es la principal fuente de la contaminación atmosférica y obteniendo emisiones diferentes debido al uso de cada combustible y tipo de vehículo usado. Saide et al. (2009) realizaron un estudio en la ciudad de Santiago de Chile de inventario de emisiones con las algunas correcciones al método proxy usado para una red de carreteras, que en conclusión subestima los resultados de emisión. El método que propone es desagregación que usa dos tipos de datos, datos indirectos (densidad de la población, uso de los suelos) y datos directos (tráfico de vehículos, contador de tráfico, capacidad de carreteras, y modelos de transporte). Ellos recomiendan usar el contador de tráfico si esto está disponible.

La comunidad Europea utiliza el método CORINAIR que considera el modelo y la categoría del vehículo, esto permite que cada país de la Comunidad Europea tenga su inventario de emisiones móviles los cuales son el punto de partida para realizar la modelación de la dispersión de contaminantes atmosféricos (Toll y Baldasano, 2000), estos inventarios son revisados y actualizados anualmente (Moreno et al., 2006). En Latinoamérica se tienen trabajos sobre el cálculo de emisiones vehiculares de contaminantes atmosféricos en la ciudad de Medellín - Colombia usando factores de emisión CORINAIR (EMEP/CORINAIR, 2007) con lo cual se realizó un inventario de emisiones de fuentes móviles. En la Habana Cuba se realizó trabajos de modelación de la 
dispersión de contaminantes atmosféricos emitidos por el tráfico vehicular en una vía utilizando sistemas de cómputo, los resultados permitieron analizar estrategias con vistas a reducir las emisiones y afectaciones generadas por el tráfico de vehículos (Paz, 2005). En Ecuador ha avanzado en lo relacionado al uso del sistema WRF-Chem para la representación del comportamiento de calidad del aire de la zona del Distrito de Quito con un inventario de emisiones propio (Parra et al., 2006). En Colombia desde 2001 la Universidad Pontifica Bolivariana ha desarrollado estudios para la generación de inventarios de emisiones y su influencia en la calidad del Aire de Medellín y el Valle de Aburra, aplicando técnicas de estimación como el modelo EUMAC zooming model (Moussiopouluos, 1995) por Toro et al. (2001), complementando sus resultados mediante la ejecución y evaluación del sistema de simulación de calidad del aire conformado por el modelo Regional Atmospheric Modeling System (Pielke et al., 1992; Cotton et al., 2003) acoplado al módulo de calidad del aire Comprehensive Air Quality Model with eXtensions (Environ, 2007) denominado RAMS-CAMx para gases y partículas (CIDI 2008).

En nuestro país en el año 2002 se realizaron trabajos de investigación sobre la contaminación ambiental generada por el tráfico de vehículos en forma conjunta por la Municipalidad Metropolitana de Lima, Asociación Peruana de Consumidores y Usuarios (ASPEC), Servicio Nacional de Adiestramiento en trabajo Industrial (SENATI) - Lima y liderados por el Ministerio de Transportes, en base a esto se ha desarrollado el Proyecto Piloto Demostrativo Ambiental "Control de Gases Contaminantes de Vehículos Motorizados" (CGC), como parte del Proyecto Piloto Demostrativo Ambiental Generación de Tecnologías en Restauración de Áreas con Pastos Alto andinos en Zonas de Explotación Minera (APGEPSENREM)/USAID, cabe indicar que este trabajo se realizó sobre la muestra de 6555 vehículos del transporte público.

En este artículo se presentan un método de inventario de emisiones que determina las emisiones de la contaminación atmosférica en Lima Metropolitana debido a que el parque automotor es el de mayor influencia de la contaminación atmosférica. Luego se realiza un estudio profundizado de las variables como número de vehículos por tipo de combustible, enfermos, costo por salud e ingreso del gas natural en la conversión del parque automotor. Es así que en búsqueda de reducir el impacto ambiental, reducir el número de enfermos, reducir el costo por salud, se realiza los cálculos de emisiones y se propone una tasa de conversión de gas natural vehicular. Por lo tanto, nuestro trabajo de investigación contribuirá primeramente a la literatura científica y que pueda ser usado en programas de modelación numérica, y a la comunidad política peruana para tomas de decisiones ambientales para la aceleración de conversión vehicular a gas natural. Para nuestro grupo de investigación permitirá simular eventos atmosféricos en Lima Metropolitana.

\section{MATERIALES Y MÉTODOS}

El estudio corresponde a la ciudad de Lima Metropolitana. Localizada a $12^{\circ} 02^{\prime} 45.29^{\prime \prime}$ Latitud Sur y $77^{\circ}$ 01 '49.97" Longitud Oeste. Su extensión es de $2672 \mathrm{~km}^{2}$, de los cuales $798,6 \mathrm{~km}^{2}$ son de área urbanizada, $179,9 \mathrm{~km}^{2}$ de tierras de cultivo y $1833,5 \mathrm{~km}^{2}$ de tierras de morfología colinosa y montañosa con pocas posibilidades de ser urbanizadas. Limita por el este con las estribaciones de la Cordillera de los Andes y por el oeste con el océano Pacífico, con una altitud de 154 metros sobre el nivel del mar en promedio.

\section{Parque automotor}

Para el análisis del parque automotor se utilizó la cantidad de vehículos que se tiene registrado según el INEI hasta el 2013, según se muestra en la Tabla 1. De acuerdo a los datos de la Tabla 1, se puede pronosticar el crecimiento vehicular de acuerdo a la ecuación exponencial. La ecuación es:

$$
y=1.80442 \mathrm{E}-39 \mathrm{e}^{0.051345138 \mathrm{x}}
$$

El coeficiente de determinación de la ecuación (1) igual a 0.9517. Esto nos indica la relación directa del comportamiento de crecimiento que tendrán los vehículos cada año.

Por otro lado, según el CIDATT, (2010) el $83 \%$ de los viajes motorizados en Lima, que equivalen aproximadamente a 10 millones de viajes, se realizan en transporte público, y de este total el $37 \%$ se realiza en combi, $30 \%$ en microbús, el $16 \%$ en ómnibus, el $9 \%$ en taxi, el $6 \%$ en moto taxi y el $2 \%$ en auto colectivo. Es necesario, tener en cuenta que el mayor usuario de la vialidad en la ciudad la tiene el taxi, estimada en $51 \%$, frente al $12 \%$ del que hace el ómnibus y camioneta rural de forma conjunta. Desde los años 90 se ha visto un incremento masivo en el transporte individual como el taxi, el auto colectivo, la moto taxis y el uso del auto privado. Para poder determinar la cantidad de flota vehicular por tipo de transporte debemos saber la cantidad de cada tipo de vehículo que conforma el parque automotor y ello se observa en la Tabla 2. 
Tabla 1: Cantidad de vehículos en Lima Metropolitana (INEI, 2016)

\begin{tabular}{|c|c|r|}
\hline $\mathrm{N}^{\circ}$ & Año & \multicolumn{1}{c|}{ Vehículos } \\
\hline 1 & 2001 & 802,748 \\
\hline 2 & 2002 & 829,214 \\
\hline 3 & 2003 & 851,360 \\
\hline 4 & 2004 & 866,881 \\
\hline 5 & 2005 & 885,636 \\
\hline 6 & 2006 & 912,763 \\
\hline 7 & 2007 & 957,368 \\
\hline 8 & 2008 & $1,036,850$ \\
\hline 9 & 2009 & $1,106,444$ \\
\hline 10 & 2010 & $1,195,353$ \\
\hline 11 & 2011 & $1,287,454$ \\
\hline 12 & 2012 & $1,395,576$ \\
\hline 13 & 2013 & $1,450,804$ \\
\hline
\end{tabular}

Tabla 2: Clasificación del parque automotor por tipo de transporte (unidad) (INEI, 2014)

\begin{tabular}{|l|c|c|c|c|c|c|c|c|c|}
\hline Año & Automóvil & $\begin{array}{l}\text { Station } \\
\text { Wagon }\end{array}$ & Ómnibus & $\begin{array}{l}\text { Camioneta } \\
\text { Rural }\end{array}$ & $\begin{array}{l}\text { Camioneta } \\
\text { Panel }\end{array}$ & $\begin{array}{l}\text { Camioneta } \\
\text { Pick Up }\end{array}$ & Camión & Remolcador & $\begin{array}{l}\text { Remolque } \\
\text { Semiremolque }\end{array}$ \\
\hline 2001 & 445,200 & 107,300 & 29,700 & 69,900 & 13,400 & 75,100 & 45,700 & 8,100 & 8,400 \\
\hline 2002 & 445,200 & 120,600 & 29,400 & 72,000 & 14,200 & 75,900 & 45,400 & 8,600 & 8,900 \\
\hline 2003 & 461,500 & 131,100 & 29,100 & 73,400 & 14,900 & 77,500 & 45,100 & 8,800 & 9,900 \\
\hline 2004 & 464,300 & 139,600 & 28,900 & 75,000 & 15,800 & 79,100 & 44,800 & 9,100 & 10,300 \\
\hline 2005 & 468,800 & 146,400 & 28,900 & 78,100 & 16,700 & 81,800 & 45,000 & 8,600 & 11,200 \\
\hline 2006 & 476,800 & 154,000 & 29,100 & 83,500 & 17,600 & 85,000 & 45,700 & 9,100 & 11,900 \\
\hline 2007 & 490,800 & 164,000 & 29,700 & 91,700 & 18,500 & 90,700 & 47,900 & 10,600 & 13,400 \\
\hline 2008 & 523,800 & 173,400 & 31,000 & 107,300 & 20,500 & 99,700 & 52,400 & 12,900 & 16,000 \\
\hline 2009 & 551,300 & 184,200 & 32,500 & 121,800 & 21,700 & 106,900 & 56,600 & 13,900 & 17,600 \\
\hline 2010 & 589,600 & 195,000 & 34,800 & 138,200 & 22,900 & 119,300 & 61,000 & 15,100 & 19,500 \\
\hline 2011 & 629,200 & 198,800 & 36,700 & 163,300 & 24,300 & 129,400 & 67,500 & 16,700 & 21,600 \\
\hline 2012 & 681,100 & 202,600 & 38,600 & 191,100 & 25,400 & 141,100 & 73,200 & 18,400 & 23,900 \\
\hline 2013 & 708,300 & 200,500 & 39,300 & 208,400 & 25,900 & 148,100 & 75,800 & 19,200 & 25,300 \\
\hline
\end{tabular}

Para este estudio el parque automotor se segmentó según el uso de combustible, los cuales se agrupan en dos grupos siendo la gasolina y el diésel y en cada grupo pertenecen los siguientes vehículos según, la Dirección General de Electricidad del Ministerio de Energía y Minas en su informe de "Elaboración de Proyectos de Guías de Orientación Del Uso Eficiente de la Energía y de Diagnóstico Energético Transporte”. En gasolina están los automóviles y station wagon; y en diésel están la camioneta panel, camioneta pick up, camión, remolcador, ómnibus, remolque y semirremolque (MINEM, 2008). A consecuencia del ingreso del proyecto Gas de Camisea (2004) en el Perú se genera un cambio en el comportamiento del parque automotor, teniendo como consecuencia la conversión de los vehículos que usan gasolina a gas natural vehicular. Es importante indicar que los combustibles a considerar (gasolina, diésel, gas licuado de petróleo y GNV) son los que conforman el parque automotor en Lima Metropolitana en el periodo de estudio de evaluación (2006 al 2013). En la Tabla 3 se registra los vehículos convertidos a GLP y GNV según se muestra la Tabla 3.

Tabla 3: Parque vehicular por tipo de combustible (unidades)

${ }^{* *}$ : Cámara Peruana de gas natural vehicular, 2015)

\begin{tabular}{|r|r|r|r|r|}
\hline & Gasolina $\left(^{*}\right)$ & Diésel $\left(^{*}\right)$ & GLP $\left(^{* *}\right)$ & GNV $\left(^{* *}\right)$ \\
\hline 2006 & 630,800 & 281,900 & 65,305 & 5,489 \\
\hline 2007 & 654,800 & 302,500 & 99,139 & 23,958 \\
\hline 2008 & 697,200 & 339,800 & 129,232 & 57,419 \\
\hline 2009 & 735,500 & 371,000 & 126,332 & 81,029 \\
\hline 2010 & 784,600 & 410,800 & 145,547 & 103,712 \\
\hline 2011 & 828,000 & 459,500 & 166,279 & 125,519 \\
\hline
\end{tabular}




\section{Factores de emisión}

Según la Organización Mundial de la Salud (OMS) en las "Guías de calidad de aire" mencionan que los cuatro contaminantes que ocasionan perjuicio a la salud son: material particulado (PM, por sus siglas en inglés), ozono $\left(\mathrm{O}_{3}\right)$, dióxido de nitrógeno $\left(\mathrm{NO}_{2}\right)$ y dióxido de azufre $\left(\mathrm{SO}_{2}\right)$; siendo los factores de emisión por el tipo de combustible de estos contaminantes por kilómetro recorrido urbano tal como se muestran en la Tabla 4 (Pérez, 2010).

Tabla 4: Factores de emisión según tipo de combustible $(\mathrm{kg} / \mathrm{km})$

\begin{tabular}{|c|c|c|c|c|}
\hline Sustancia & Gasolina & Diésel & GLP & GNV \\
\hline Monóxido de Carbono & 0.000984000 & 0.000610400 & 0.0004465000 & 0.0004465000 \\
\hline Óxidos de Nitrógeno & 0.000051300 & 0.000518000 & 0.0000206400 & 0.0000206400 \\
\hline Material Particulado menor a $2.5 \mu \mathrm{m}$ & 0.000008480 & 0.000037100 & 0.0000073670 & 0.0000073670 \\
\hline Material Particulado mayor a $10 \mu \mathrm{m}$ & 0.000078000 & 0.000079300 & 0.0000780000 & 0.0000780000 \\
\hline Material Particulado mayor a 2.5 um y menor a $10 \mu \mathrm{m}$ & 0.000013550 & 0.000015940 & 0.0000134000 & 0.0000134000 \\
\hline Dióxido de Azufre & 0.000006034 & 0.000005540 & 0.0000009412 & 0.0000009412 \\
\hline
\end{tabular}

\section{Enfermos por vías respiratorias}

Los enfermos por afecciones respiratorias se muestran en la Tabla 5. Los datos están disponibles en un reporte del Instituto Nacional de Estadística e Informática INEI, a partir del año 2006.

Tabla 5: Cantidad de enfermos por afecciones respiratorias al año

\begin{tabular}{|l|c|}
\hline Año & Afecciones respiratorias \\
\hline 2006 & $3,330,185$ \\
\hline 2007 & $3,876,350$ \\
\hline 2008 & $3,902,028$ \\
\hline 2009 & $3,927,705$ \\
\hline 2010 & $4,176,790$ \\
\hline 2011 & $4,208,090$ \\
\hline
\end{tabular}

\section{Metodología}

La metodología usada para las proyecciones serán los modelos estadísticos proyectados en la actualidad y propuestos del 2014 al 2040 según se puede visualizar en la Figura 1.

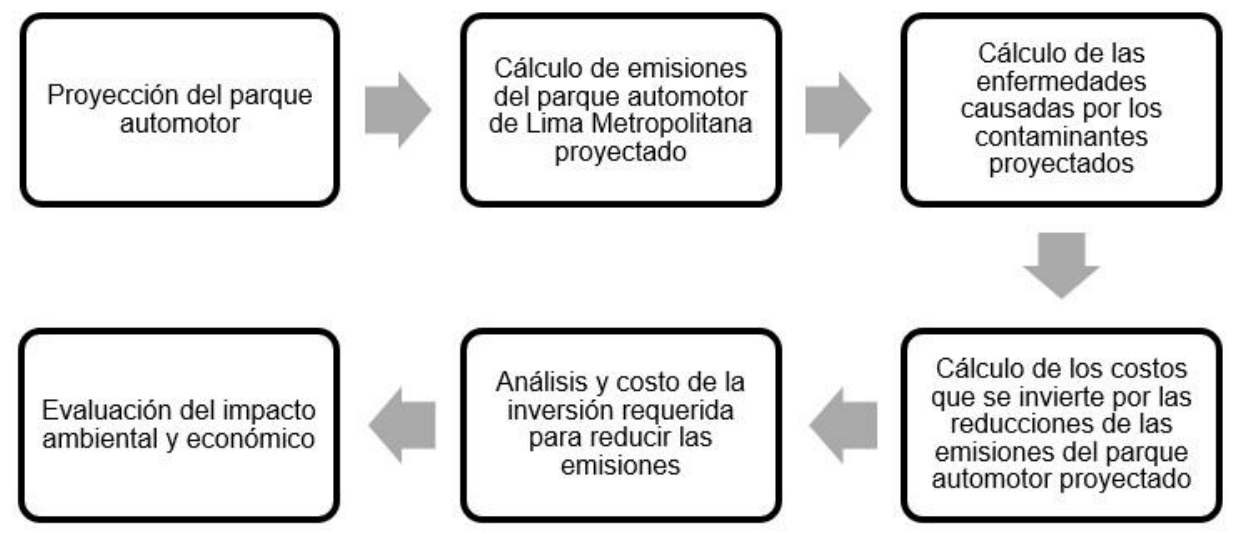

Fig. 1: Metodología de cálculo para el impacto económico desarrollado

\section{RESULTADOS Y DISCUSIÓN}

Como se puede visualizar en la Tabla 6 se presenta el total de emisiones calculadas mediante los factores de

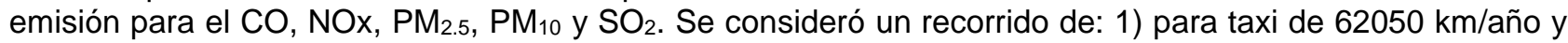
2) para auto privado de $21900 \mathrm{~km} / \mathrm{año}$.

En la Tabla 7 se muestra la cantidad de personas con afecciones respiratorias (ne), costo de salud (Cs) y costo de inasistencia laboral (Ci) proyectadas al 2040, los costos de salud por enfermedad anual se calculan según la ecuación (3) y el costo de inasistencia laboral anual por enfermedad según la ecuación (4). Considerando el ingreso diario (id) $=61$ soles. 
Tabla 6: Total de emisiones por contaminante (kg/año)

\begin{tabular}{|c|c|c|c|c|c|}
\hline Año & CO & NOx & $P M_{2.5}$ & $P M_{10}$ & $\mathrm{SO}_{2}$ \\
\hline 2006 & $40,817,872$ & $10,631,269$ & 909,898 & $1,826,628$ & 281,120 \\
\hline 2007 & $40,594,146$ & $11,238,029$ & 952,701 & $1,996,509$ & 279,986 \\
\hline 2008 & $41,801,131$ & $12,420,717$ & $1,044,101$ & $2,326,395$ & 287,816 \\
\hline 2009 & $44,578,921$ & $13,502,890$ & $1,134,836$ & $2,607,158$ & 305,696 \\
\hline 2010 & $47,100,471$ & $14,831,295$ & $1,240,150$ & $2,918,090$ & 323,067 \\
\hline 2011 & $49,595,277$ & $16,426,938$ & $1,362,626$ & $3,263,900$ & 341,364 \\
\hline 2012 & $79,540,983$ & $28,914,899$ & $2,342,465$ & $5,390,504$ & 562,391 \\
\hline 2013 & $85,033,606$ & $31,763,639$ & $2,563,259$ & $5,950,311$ & 602,809 \\
\hline 2014 & $91,104,256$ & $34,908,198$ & $2,806,433$ & $6,556,820$ & 647,753 \\
\hline 2015 & $97,792,740$ & $38,378,132$ & $3,074,131$ & $7,214,586$ & 697,570 \\
\hline 2016 & $105,144,618$ & $42,206,136$ & $3,368,732$ & $7,928,658$ & 752,650 \\
\hline 2017 & $113,211,272$ & $46,428,435$ & $3,391,184$ & $8,585,293$ & 768,626 \\
\hline 2018 & $122,049,949$ & $51,084,906$ & $4,049,467$ & $9,548,685$ & 880,406 \\
\hline 2019 & $131,724,195$ & $56,219,711$ & $4,441,734$ & $10,467,640$ & 954,110 \\
\hline 2020 & $142,303,847$ & $61,881,408$ & $4,873,218$ & $11,469,011$ & $1,035,139$ \\
\hline 2021 & $153,866,157$ & $68,123,742$ & $5,347,842$ & $9,171,520$ & $1,124,151$ \\
\hline 2022 & $166,495,866$ & $75,005,913$ & $5,869,923$ & $9,822,230$ & $1,221,867$ \\
\hline 2023 & $180,285,995$ & $82,593,257$ & $6,444,224$ & $10,521,903$ & $1,329,081$ \\
\hline 2024 & $195,338,800$ & $90,957,834$ & $7,075,996$ & $11,274,189$ & $1,446,668$ \\
\hline 2025 & $211,766,297$ & $100,179,094$ & $7,771,026$ & $12,083,057$ & $1,575,587$ \\
\hline 2026 & $229,691,497$ & $110,344,750$ & $8,535,697$ & $12,952,831$ & $1,716,891$ \\
\hline 2027 & $249,249,351$ & $121,551,494$ & $9,377,045$ & $13,888,225$ & $1,871,740$ \\
\hline 2028 & $270,587,598$ & $133,905,978$ & $10,302,822$ & $14,894,347$ & $2,041,406$ \\
\hline 2029 & $293,868,676$ & $147,525,876$ & $11,321,581$ & $15,976,785$ & $2,227,289$ \\
\hline 2030 & $319,270,566$ & $162,540,985$ & $12,442,748$ & $17,141,605$ & $2,430,924$ \\
\hline 2031 & $346,988,405$ & $179,094,425$ & $13,676,711$ & $18,395,402$ & $2,654,001$ \\
\hline 2032 & $377,236,591$ & $197,344,169$ & $15,034,932$ & $19,745,375$ & $2,898,376$ \\
\hline 2033 & $410,250,101$ & $217,464,375$ & $16,530,038$ & $21,199,345$ & $3,166,089$ \\
\hline 2034 & $446,287,024$ & $239,647,148$ & $18,175,955$ & $22,765,849$ & $3,459,383$ \\
\hline 2035 & $485,630,586$ & $264,104,518$ & $19,988,040$ & $24,454,172$ & $3,780,724$ \\
\hline 2036 & $528,591,786$ & $291,070,296$ & $21,983,226$ & $26,274,444$ & $4,132,822$ \\
\hline 2037 & $575,511,986$ & $320,802,350$ & $24,180,176$ & $28,237,706$ & $4,518,659$ \\
\hline 2038 & $626,766,211$ & $353,585,281$ & $26,599,479$ & $30,355,994$ & $4,941,515$ \\
\hline 2039 & $682,766,422$ & $389,732,948$ & $29,263,835$ & $32,642,453$ & $5,404,994$ \\
\hline 2040 & $743,965,004$ & $429,591,635$ & $32,198,275$ & $35,111,407$ & $5,913,066$ \\
\hline
\end{tabular}

Para determinar el costo que genera el total de emisiones por contaminante se determinó la cantidad de personas con afecciones respiratorias hasta el 2040. La regresión es lineal con un coeficiente de determinación $\mathrm{R}^{2}$ de 0.811678 (ecuación 2).

$$
y=151900.6286 x-301188887.8
$$

Luego se consideró determinar el costo por salud (ecuación 3) y otro por inasistencia (ecuación 4). Para determinar las veces que se enferma al año la población limeña se realizó una encuesta personal a la población por enfermarse por afecciones respiratorias anualmente (500 personas entrevistadas). Los resultados de esta encuesta lograron determinar que las personas limeñas se enferman y dejan de asistir a su labor de trabajo un promedio de tres veces al año. Se consideró el gasto por salud promedio de 50 soles (situaciones de alergia, gripe y resfríos excepto las situaciones crónicas).

$$
\begin{aligned}
& \mathrm{Cs}=\text { ne }{ }^{*} \text { gsp * vea } \\
& \mathrm{Ci}=\text { ne * id * vea }
\end{aligned}
$$


Donde "Cs" es el costo por salud, "Ci" es el costo por inasistencia, "ne" el número de enfermos, "gsp" el gasto de salud promedio, "vea" a las veces que se enferma al año, e "id" es el ingreso diario.

Tabla 7: Personas con afecciones respiratorias, costo de salud y costo de inasistencia laboral al 2040.

\begin{tabular}{|r|r|r|r|}
\hline Año & $\begin{array}{c}\text { Afecciones } \\
\text { respiratorias }\end{array}$ & $\begin{array}{c}\text { Costo de salud } \\
\text { (soles) }\end{array}$ & $\begin{array}{c}\text { Costo de } \\
\text { inasistencia } \\
\text { laboral (soles) }\end{array}$ \\
\hline 2006 & $3,330,185$ & & \\
\hline 2007 & $3,876,350$ & & \\
\hline 2008 & $3,902,028$ & & \\
\hline 2009 & $3,927,705$ & & \\
\hline 2010 & $4,176,790$ & & \\
\hline 2011 & $4,208,090$ & & \\
\hline 2012 & $4,435,177$ & & \\
\hline 2013 & $4,587,077$ & & $901,002,575$ \\
\hline 2014 & $4,738,978$ & & $929,490,138$ \\
\hline 2015 & $4,890,879$ & $733,631,813$ & \\
\hline 2016 & $5,042,779$ & $756,416,907$ & \\
\hline 2017 & $5,194,680$ & $779,202,001$ & $958,008,082$ \\
\hline 2018 & $5,346,581$ & $801,987,096$ & $986,556,406$ \\
\hline 2019 & $5,498,481$ & $824,772,190$ & $1,015,135,110$ \\
\hline 2020 & $5,650,382$ & $847,557,284$ & $1,043,744,194$ \\
\hline 2021 & $5,802,283$ & $870,342,379$ & $1,072,383,658$ \\
\hline 2022 & $5,954,183$ & $893,127,473$ & $1,101,053,503$ \\
\hline 2023 & $6,106,084$ & $915,912,567$ & $1,129,753,727$ \\
\hline & & & \\
\hline
\end{tabular}

\begin{tabular}{|r|r|r|c|}
\hline Año & $\begin{array}{c}\text { Afecciones } \\
\text { respiratorias }\end{array}$ & $\begin{array}{c}\text { Costo de salud } \\
\text { (soles) }\end{array}$ & $\begin{array}{c}\text { Costo de } \\
\text { inasistencia } \\
\text { laboral (soles) }\end{array}$ \\
\hline 2024 & $6,257,984$ & $938,697,661$ & $1,158,484,332$ \\
\hline 2025 & $6,409,885$ & $961,482,756$ & $1,187,245,317$ \\
\hline 2026 & $6,561,786$ & $984,267,850$ & $1,216,036,682$ \\
\hline 2027 & $6,713,686$ & $1,007,052,944$ & $1,244,858,427$ \\
\hline 2028 & $6,865,587$ & $1,029,838,039$ & $1,273,710,552$ \\
\hline 2029 & $7,017,488$ & $1,052,623,133$ & $1,302,593,057$ \\
\hline 2030 & $7,169,388$ & $1,075,408,227$ & $1,331,505,942$ \\
\hline 2031 & $7,321,289$ & $1,098,193,321$ & $1,360,449,208$ \\
\hline 2032 & $7,473,189$ & $1,120,978,416$ & $1,389,422,854$ \\
\hline 2033 & $7,625,090$ & $1,143,763,510$ & $1,418,426,879$ \\
\hline 2034 & $7,776,991$ & $1,166,548,604$ & $1,447,461,285$ \\
\hline 2035 & $7,928,891$ & $1,189,333,699$ & $1,476,526,071$ \\
\hline 2036 & $8,080,792$ & $1,212,118,793$ & $1,505,621,237$ \\
\hline 2037 & $8,232,693$ & $1,234,903,887$ & $1,534,746,784$ \\
\hline 2038 & $8,384,593$ & $1,257,688,981$ & $1,563,902,710$ \\
\hline 2039 & $8,536,494$ & $1,280,474,076$ & $1,593,089,017$ \\
\hline 2040 & $8,688,394$ & $1,303,259,170$ & $1,622,305,703$ \\
\hline
\end{tabular}

Se propone, entonces la conversión del parque automotor de gasolina a gas natural vehicular en una mayor tendencia a la actual (Tabla 8). La propuesta es la de aumentar el volumen del parque automotor convertido a gas natural en el doble del promedio de la variación del incremento actual (3\%). Es decir, la propuesta es de incrementar en un $6 \%$ anual la conversión a gas natural. Y según la propuesta realizada se redujo la contaminación de $\mathrm{CO}, \mathrm{NOx}, \mathrm{MP}_{2.5}$, PTS, $\mathrm{PM}_{10}$ y SO 2 según se puede visualizar en la Figura 2 y 3 . La reducción del total de enfermos se puede visualizar en la Figura 4. Y la reducción de costo de salud se puede visualizar en la Figura 5. La reducción de costo por inasistencia se muestra en la Figura 6.

Tabla 8: Porcentaje de conversión de gasolina a gas natural vehicular actual.

\begin{tabular}{|c|c|}
\hline Año & $\begin{array}{c}\text { Porcentaje de Conversión } \\
\text { (Gasolina a Gas Natural) }\end{array}$ \\
\hline 2006 & $0.810 \%$ \\
\hline 2007 & $3.349 \%$ \\
\hline 2008 & $7.525 \%$ \\
\hline 2009 & $9.962 \%$ \\
\hline 2010 & $12.538 \%$ \\
\hline 2011 & $14.594 \%$ \\
\hline
\end{tabular}

Los resultados del trabajo concuerdan en los temas de salud con investigaciones desarrolladas por ejemplo de Placeres et al. (2006) sobre la influencia de la contaminación en la salud de las personas, en la india con la investigación de Knote et al. (2018) donde el $\mathrm{PM}_{2.5}$ contribuye sobre las enfermedades respiratorias y con estudios de Maji et al. (2016) sobre la morbilidad debido al PM10. También concuerdan en temas de emisiones con investigaciones de Zhang (2018) que el inventario de emisiones tiene que ser actualizado constantemente para realizar un buen pronóstico, con la investigación de Huo (2011) que las emisiones están relacionadas al tipo de vehículo y combustible. 
Por otro lado, en temas de sectores predominantes de emisiones también concuerda que es el parque automotor quien predomina como mayor portador de las emisiones tal como lo mencionan Londoño et al. (2011) y otros investigadores como Wang et al. (2008). Si bien hay muchas similitudes con otras investigaciones al concluir que el inventario de emisiones es muy importante para pronósticos de calidad de aire, la diferencia está en que en este trabajo se presentan las relaciones con otras variables incorporando la estrategia de aumentar la conversión del parque automotor a gas natural, consiguiéndose una reducción de enfermos, reducción de costo por salud y además reducción de costos por inasistencia a una proyección al 2040.

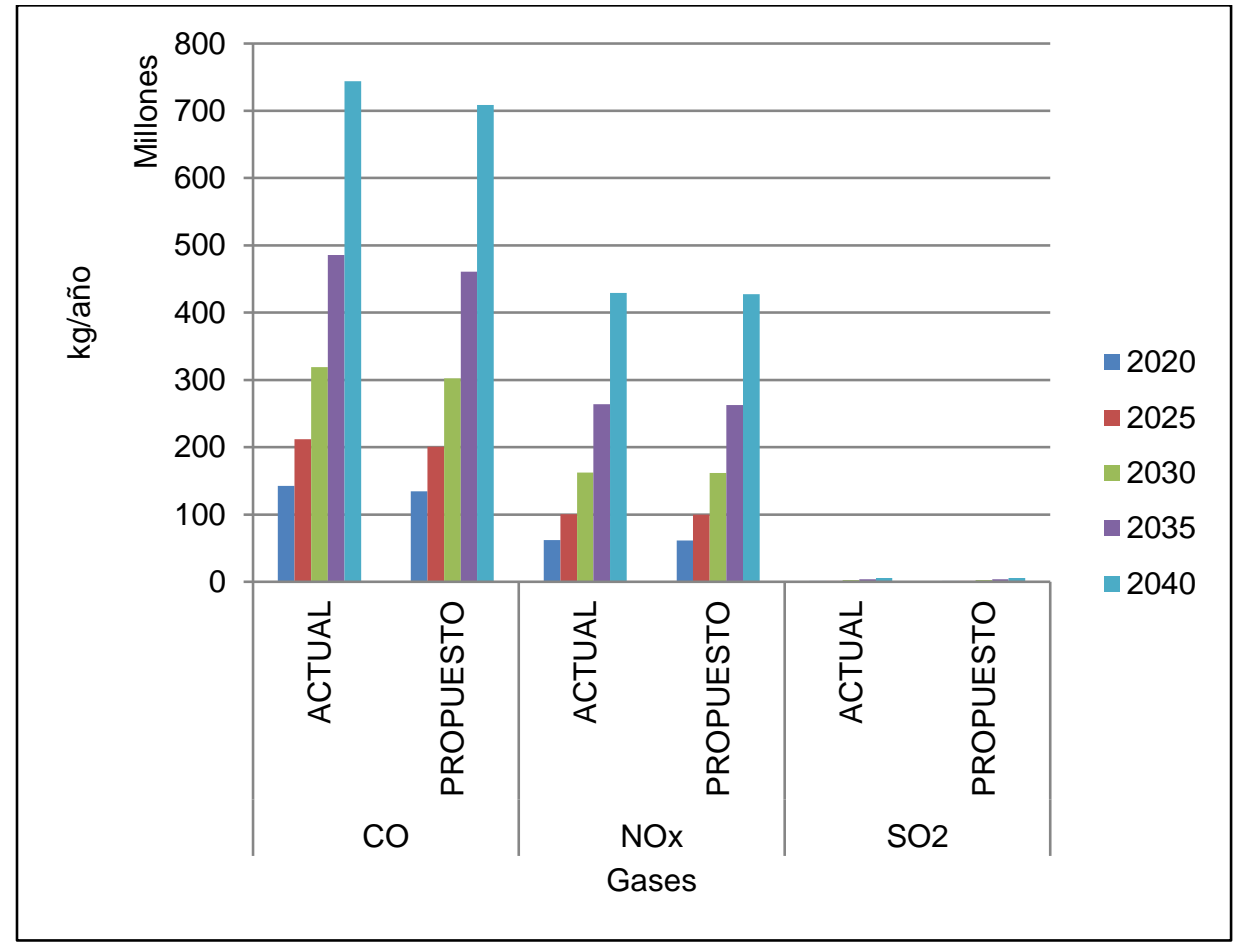

Fig. 2: Reducción de gases contaminantes $\left(\mathrm{CO}, \mathrm{NOx}, \mathrm{SO}_{2}\right)$

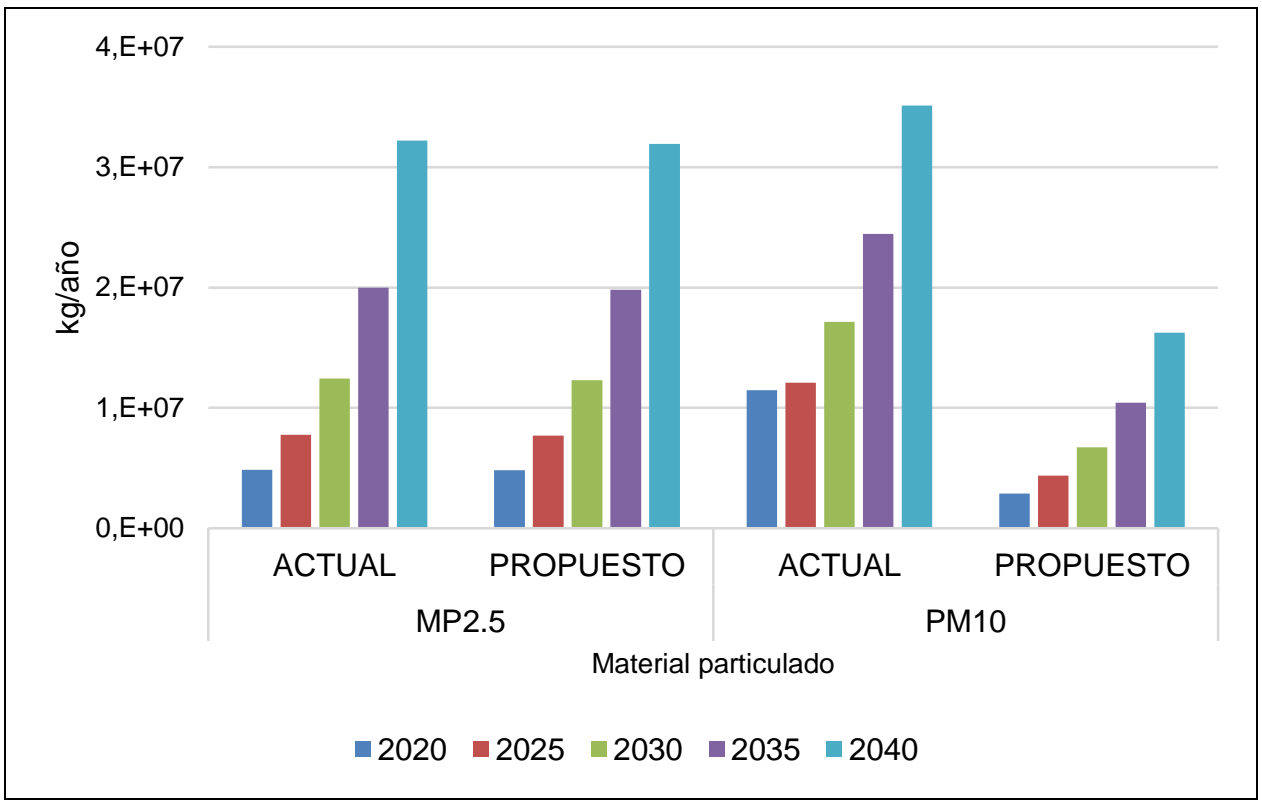

Fig. 3: Reducción de $\mathrm{PM}_{2.5}$ y $\mathrm{PM}_{10}$ 


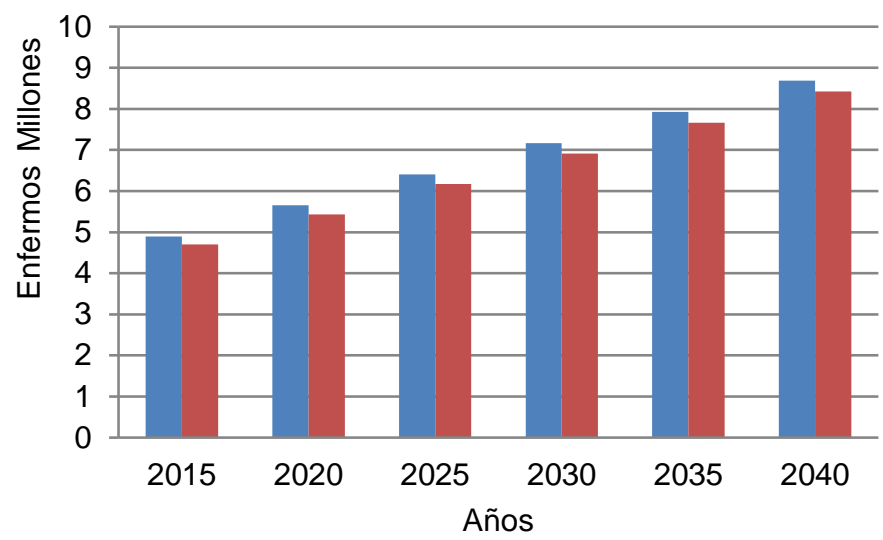

- Actual

- Propuesto

Fig. 4: Reducción de enfermos

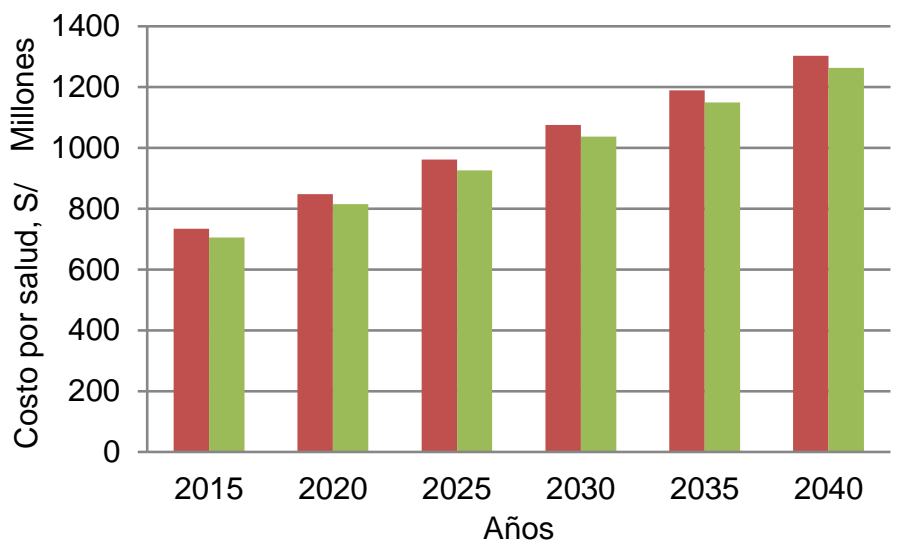

- Actual

- Propuesto

Fig. 5: Reducción de costo por salud

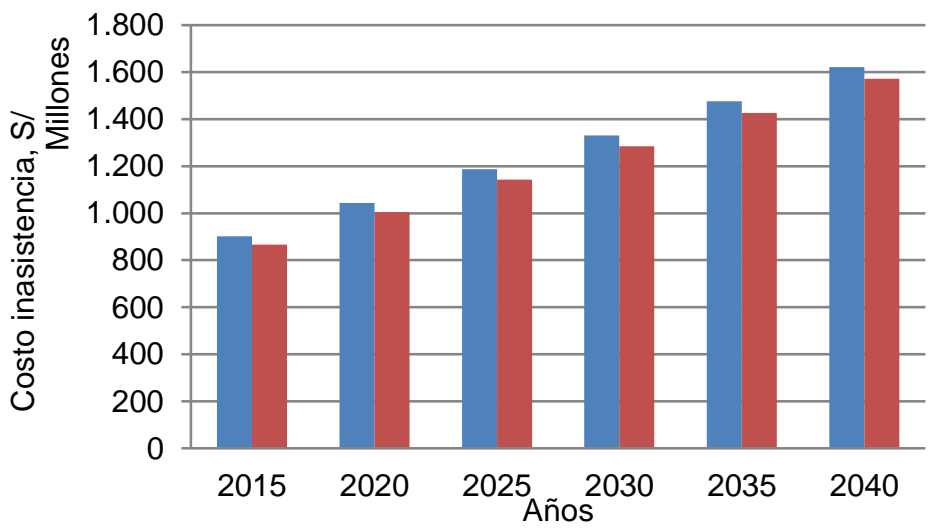

- Actual

- Propuesto

Fig. 6: Reducción de costos por inasistencia

\section{CONCLUSIONES}

Se concluye que los resultados esperados coinciden con los resultados de otros investigadores en temas de predominio del sector automotor, influencia de la contaminación con temas de salud, enfermedades respiratorias y morbilidad debido al $\mathrm{PM}_{2.5}$ y $\mathrm{PM}_{10}$, y la importancia de un inventario de emisiones actualizado para realizar un buen pronóstico. Existe una gran oportunidad de reducir las emisiones de contaminantes, al realizar el cambio de combustible de los vehículos por gas natural. En este estudio se simula el efecto de un aumento, mínimo, de $3 \%$ adicional anual, lográndose reducir un promedio de $5 \%(\mathrm{CO}), 0.5 \%$ (NOx) y $4.5 \%$ $\left(\mathrm{SO}_{2}\right)$ y $62 \%$ de material particulado $\mathrm{PM}_{10}$. También se logra una reducción de la cantidad de personas con enfermedades respiratorias, en un 3.6\%. Paralelamente, se lograría una reducción en los costos de salud (tratamiento de las enfermedades respiratorias) y pérdidas por inasistencia (por descanso médico) en un $3.6 \%$. Finalmente, los avances actuales muestran que, si es posible disminuir las emisiones gaseosas y material particulado en Lima Metropolitana por el uso del gas natural en la matriz energética del país, reducir la cantidad de personas enfermas y reducir los costos asociados por salud si se realiza una acción proactiva en la conversión del parque automotor a gas natural vehicular en Lima Metropolitana. 


\section{AGRADECIMIENTOS}

El autor y colaboradores agradecen a la Pontificia Universidad Católica del Perú, Universidad Nacional Agraria La Molina y SENAMHI quienes han apoyado a la investigación.

\section{REFERENCIAS}

Banco Mundial, Análisis Ambiental del Perú: Retos para un desarrollo sostenible. Análisis Ambiental del Perú, Lima (2007)

Conibear, L., E.W. Butt y otros 3 autores, Residential energy use emissions dominate health impacts from exposure to ambient particulate matter in India, doi: 10.1038/s41467-018-02986-7, Nature Communications, 9(1), 1-9 (2018)

Huo, H., Q. Zhang y otros tres autores, Modeling vehicle emissions in different types of Chinese cities: Importance of vehicle fleet and local features, doi: 10.1016/j.envpol.2011.04.025, Environmental Pollution, 159(10), 2954-2960 (2011)

INEI, Perú: Anuario de Estadísticas Ambientales 2014, 574 (2014)

INEI, Compendio estadístico Perú 2016 Tomo II, 2(Cnpv 2012), 949-1790 (2016)

Londoño, J., M.A. Correa y C.A. Palacio, Estimación de las emisiones de contaminantes atmosféricos provenientes de fuentes móviles en el área urbana de Envigado, Colombia, Revista EIA., (16), 149-162 (2011)

Maji, K. J., A.K. Dikshit, A. Deshpande y P.C. Speldewinde, Human health risk assessment due to air pollution in ten urban cities in Maharashtra, India, doi: 10.1080/23311843.2016.1193110, Cogent Environmental Science, 11(2), 0 (2016)

Minsa, Dirección de Salud V Lima Ciudad 2012, Lima (2012)

Moreno, J. R., J. David y otros tres autores, Estimación de la emisión de contaminantes debida al tráfico urbano mediante modelos de asignación de tráfico (2006)

Parra, R., P. Jiménez y J.M. Baldasano, Development of the high spatial resolution EMICAT2000 emission model for air pollutants from the north-eastern Iberian Peninsula (Catalonia, Spain), doi: 10.1016/j.envpol.2005.07.021, Environmental Pollution, 140(2), 200-219 (2006)

Pérez, C., Tesis PUCP, Propuesta de conversión del parque automotor de Lima y Callao para el uso de gas natural, Pontificia Universidad Católica del Perú, doi: 10.1017/CBO9781107415324.004 (2010)

Placeres, M. R., F.D. Olite y M.A. Toste, La contaminación del aire: Su repercusión como problema de salud, Revista Cubana de Higiene y Epidemiologia, 44(2), 1-14 (2006)

Saide, P., R. Zah, M. Osses y M. Ossés de Eicker, Spatial disaggregation of traffic emission inventories in large cities using simplified top-down methods, doi: 10.1016/j.atmosenv.2009.07.013, Atmospheric Environment, 43(32), 4914-4923, (2009)

SENAMHI, Evaluación de la calidad del aire en Lima Metropolitana 2011, Lima (2012)

Toll, I., y J.M. Baldasano, Modeling of photochemical air pollution in the Barcelona area with highly disaggregated anthropogenic and biogenic emissions, doi: 10.1016/S1352-2310(99)00498-7, Atmospheric Environment, 34(19), 30693084 (2000)

Toro, M., J. Ramírez, R. Quiceno y C. Zuluaga, Cálculo de la emisión vehicular de contaminantes atmosféricos en la ciudad de Medellín mediante factores de emisión Corinair. Revista Acodal, (191), 42-49 (2001)

Wang, H., C. Chen, C. Huang y L. Fu, On-road vehicle emission inventory and its uncertainty analysis for Shanghai, China, doi: 10.1016/j.scitotenv.2008.01.038, Science of the Total Environment, 398(1-3), 60-67 (2008)

Zhang, L., T. Zhao y otros cinco autores, Updated emission inventories of power plants in simulating air quality during haze periods over East China, doi: 10.5194/acp-18-2065-2018, Atmospheric Chemistry and Physics, 18(3), 2065-2079 (2018) 